\title{
Terahertz modulation of the Faraday effect by laser pulses via the optical Kerr effect
}

\author{
R.R. Subkhangulov, ${ }^{1}$ R.V. Mikhaylovskiy, ${ }^{1}$ A.K. Zvezdin, ${ }^{2,3,4}$ V.V. Kruglyak, ${ }^{5}$ Th. Rasing, ${ }^{1}$ and A.V. Kimel ${ }^{1,2}$ \\ ${ }^{1)}$ Radboud University, Institute for molecules and materials, Heyendaalseweg 135 6525AJ, Nijmegen, \\ The Netherlands \\ ${ }^{2)}$ Moscow State Technical University of Radio Engineering, Electronics and Automation, 119454 Moscow, \\ Russia \\ 3) Prokhorov General Physics Institute of the Russian Academy of Sciences, 119991 Moscow, \\ Russia \\ ${ }^{4)}$ Moscow Institute of Physics and Technology (State University), 141700 Dolgoprudny, \\ Russia \\ ${ }^{5)}$ School of Physics, University of Exeter, Stocker Road, Exeter, EX4 4QL, United Kingdom
}

The magneto-optical Faraday effect, played a crucial role in the elucidation of the electromagnetic nature of light. Today it is powerful means to probe magnetism and the basic operational principle of magneto-optical modulators. Understanding the mechanisms allowing for modulation of the of magneto-optical response at $\mathrm{THz}$ frequencies may have far reaching consequences for photonics $^{1}$, the ultrafast optomagnetism ${ }^{2-4}$, magnonics $^{5,6}$ as well as for future development of ultrafast Faraday modulators. Here we suggest a conceptually new approach for an ultrafast tunable magneto-optical modulation with the help of counter propagating laser pulses. Using terbium gallium garnet $\left(\mathrm{Tb}_{3} \mathrm{Ga}_{5} \mathrm{O}_{12}\right)$ we demonstrate the feasibility of such a magneto-optical modulation with a frequency up to $1.1 \mathrm{THz}$, continuously tunable by means of an external magnetic field. Besides the novel concept for ultrafast magnetooptical polarization modulation, our findings reveal the importance of accounting for propagation effects in the interpretation of pump-probe magneto-optical experiments.

For light propagating along the $z$ axis through a homogeneous and isotropic medium the Faraday polarization rotation $\Theta_{0}$ is defined as:

$$
\Theta_{0}=d \cdot \frac{\pi \cdot \alpha \cdot M_{z}\left(B_{0}\right)}{\lambda \cdot n_{0}},
$$

where $d$ is the effective travel distance of light inside the medium, $\lambda$ is the wavelength of light in vacuum, $n_{0}$ is the refractive index at this wavelength, $M_{z}$ is the magnetization along the $z$-axis at the applied magnetic field $B_{0}$ and $\alpha$ is a magneto-optical constant of the medium. In paramagnetic and diamagnetic media the Faraday effect is described in terms of the Verdet constant defined as $V=\pi \cdot \alpha \cdot \chi /\left(\lambda \cdot n_{0}\right)$, where $\chi$ is the magnetic susceptibility so that $\Theta_{0}=d \cdot V \cdot B_{0}$. The ultrafast modulation of the Faraday effect was demonstrated using THz magnetic fields ${ }^{7-9}$ and optical excitation of $\mathrm{THz}$ oscillations of magnetization $M_{z} \cdot{ }^{10,11}$

It was shown that the Faraday effect can be enhanced $^{12,13}$ in the magneto-optical medium with the introduced inhomogeneity of the complex refractive in$\operatorname{dex} \tilde{n}=n+i \kappa$ along the $z$ direction of light propagation. The Faraday effect can be modulated in time, if the inhomogeneity is intrinsically nonstationary and moving in the medium. The laser induced acoustic solitons are an example of the propagating optical inhomogeneities created by a pulsed stress. ${ }^{14-17}$ In this case, the frequency of the Faraday modulation $\Omega$ is determined by the speed of sound and resides in a $\mathrm{GHz}$ range. An inhomogeneity moving with a relativistic speed can be created by an intense laser pulse, which due to the optical Kerr effect, induces a linear dichroic region. ${ }^{18}$ Employing the inhomogeneities moving with the group velocity of the laser pulse should result in much higher modulation frequencies.

In order to test this approach, we performed timeresolved magneto-optical studies in the Faraday geometry, in which an intense femtosecond laser pulse (pump) with a central photon energy of $1.55 \mathrm{eV}$ propagates through and interacts with a medium. The much weaker pulse (probe) interacts with the medium excited by the pump. Employing the linearly polarized pulses with duration $\Delta=70 \mathrm{fs}$, we measured the temporal evolution of the pump induced Faraday rotation and transient tran- 
missivity of the probe pulses as sketched in Fig. 1 (a) (see also Methods). We chose a (111) cut terbium gallium garnet $\left(\mathrm{Tb}_{3} \mathrm{Ga}_{5} \mathrm{O}_{12}\right)$ single crystal with a thickness $L=1 \mathrm{~mm}$. $\mathrm{Tb}_{3} \mathrm{Ga}_{5} \mathrm{O}_{12}$ is a building block of most magneto-optical modulators and optical isolators since it exhibits one of the highest Faraday rotations ${ }^{19}$ and a high transparency in the visible spectral range. ${ }^{20,21}$ The scheme of the electronic structure of the $\mathrm{Tb}^{3+}$ ion and the states of the ground multiplet ${ }^{7} \mathrm{~F}_{6}$ in $\mathrm{Tb}_{3} \mathrm{Ga}_{5} \mathrm{O}_{12}$ have been thoroughly studied ${ }^{21}$ and are shown schematically in Fig. 1 (b). The main magnetic resonances are expected to be due to the lowest states of the ground state multiplet ${ }^{7} \mathrm{~F}_{6}$ as shown schematically in Fig 1 (b). The photon energies of the probe pulse were $1.24 \mathrm{eV}, 2.48 \mathrm{eV}$ and $3.1 \mathrm{eV}$ (see all photon energies with respect to the electronic structure of the $\mathrm{Tb}^{3+}$ ion in Fig. 1 (b)).

Figure 2 (a) demonstrates a typical time dependence of the pump induced Faraday rotation and transient transmissivity of the probe with a photon energy of $3.1 \mathrm{eV}$ (a wavelength of $0.4 \mu \mathrm{m}$ ) at $B_{0}=5 \mathrm{kG}$ and temperature $T=1.7 \mathrm{~K}$. It is seen that the Faraday rotation discloses oscillations which persist only in a limited time window: at a time delay of $\Delta \tau \approx 13$ ps the signal is abruptly quenched.

We found that by pumping the sample with circularly polarized pulses the paramagnetic resonance 22,23 can be excited (Supplementary materials B). Note that while the high frequency oscillations abruptly quench at $\Delta \tau=13 \mathrm{ps}$, the oscillations corresponding to the paramagnetic resonance in $\mathrm{Tb}^{3+}$ last for a much longer time delay (Supplementary materials B). Furthermore the fast Fourier transform (FFT) spectrum in Fig. 2 (b) of the signal shows that the dominating oscillation frequency cannot be attributed to either the pump induced coherence between levels of the ground multiplet ${ }^{7} \mathrm{~F}_{6}$ of the $\mathrm{Tb}^{3+}$ ion (1.36 and $2.25 \mathrm{THz}$ ) or phonon modes (5.1 and $5.4 \mathrm{THz}) \cdot{ }^{21,24-26}$ Note that the highest frequency observed in the experiment is about 7.27 THz (Supplementary materials $\mathrm{G}$ ) indicating that the temporal resolution is at least $140 \mathrm{fs}$. This is much shorter than the pulse duration, which can be mistakingly deduced from the overlap in Fig. 2 (a) if propagation effects are not taken into account (Methods). The quenched oscillations cannot be assigned to any of the transitions within the ground multiplet ${ }^{7} \mathrm{~F}_{6}$ of the $\mathrm{Tb}^{3+}$ ion in Fig. 1 (c). ${ }^{21,27}$

To elucidate the nature of the quenched oscillations we measured the Faraday rotation of the transmitted probe for different magnetic fields at $T=1.7 \mathrm{~K}$ (Fig. 3 (a)). The quench was always observed at the time delay of $\Delta \tau \approx 13 \mathrm{ps}$ independently of magnetic field. The aforementioned oscillations dominated the signal. Their frequency varies from $0.1 \mathrm{THz}$ to $1.1 \mathrm{THz}$ with increasing the magnetic field from $100 \mathrm{G}$ to $70 \mathrm{kG}$ (Fig. 3 (b) and Supplementary materials A). It is remarkable that if these oscillations originated from a spin resonance, such a frequency change would correspond to an enormous effective $g$-factor $g_{\text {eff }}=55$. From Fig. 3 (b) and Supplementary material B it can be inferred that the frequency shows a completely different dependence on magnetic field and has a much larger effective $g$-factor in comparison with that associated with the electronic transition in the ground multiplet of the $\mathrm{Tb}^{3+}$ ions. ${ }^{22,23,28}$ In fact, the magnetic field dependence of the frequency scales as the sample magnetization.

Figure $3(\mathrm{c}, \mathrm{d})$ shows the temperature dependence of the oscillation frequency measured at $B_{0}=30 \mathrm{kG}$. A strong decrease from $1.1 \mathrm{THz}$ to $0.1 \mathrm{THz}$ upon a temperature increase from $1.7 \mathrm{~K}$ to $100 \mathrm{~K}$ is observed. If the modulation frequency is proportional to the magnetization, it has to follow the Curie-Weiss law, i.e. be inversely proportional to the ambient temperature. A linear fit of the inverse frequency as a function of temperature (inset in Fig. $3(\mathrm{~d})$ ) yielded the Curie-Weiss parameter $\Theta_{C W}=-8.2 \mathrm{~K}$, which matches well with that reported earlier. ${ }^{27}$ All these observations support a linear relation between the oscillation frequency and the net magnetization of the medium.

The origin of the large $\mathrm{THz}$ oscillations is further elucidated in measurements of the their frequency at several probe wavelengths $\lambda$ (Fig. $3(\mathrm{e}, \mathrm{f})$ ). The dependence of the oscillations on the probe wavelength is a clear signature of inhomogeneous excitation and light propagation effects $^{14,29,30}$ (see Supplementary materials D).

To explain the observed oscillations, we propose the model of a counter propagating dichroic region induced by the pump via the optical Kerr effect. If the probe pulse is behind the pump, then the part of the pump reflected from the second face of the crystal can counter propagate through the probe which via the optical Kerr effect acquires polarization rotation. ${ }^{18}$

If the proposed interpretation is true, the observed modulation of the Faraday rotation has to be quenched as soon as the time delay between the two pulses becomes so long that the probe pulse cannot encounter with the 
counter propagating pump any longer. Taking into account the group refractive index of the probe $n_{p r}$ and the pump $n_{p u}$ in $\mathrm{Tb}_{3} \mathrm{Ga}_{5} \mathrm{O}_{12}$ (Supplementary material $\mathrm{F}$ ), we calculated the maximum time delay $\Delta \tau$ at which the probe can still interact with the counter propagating pump via the optical Kerr effect (Methods):

$$
\Delta \tau=\frac{2 L \cdot n_{p u}}{c_{0}} .
$$

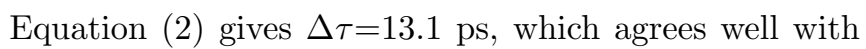
the time at which signal is abruptly quenched in the experiment (Fig. $3(\mathrm{e})$ ). A slight deviations from this time are due to small noncollinearity of the pump with respect to the sample normal.

The concept as shown in Fig. 4 accounting for the interaction between the probe and the reflected counter-propagating pump anticipates that the transient magneto-optical response should oscillate with the frequency (Methods)

$$
\Omega=\frac{2 B_{0} c_{0} V_{+}}{\left(n_{p u}+n_{p r}\right)},
$$

where $V_{+}=V_{p u}+V_{p r}$, with $V_{p u}, V_{p r}$ being the Verdet constants at wavelength of the pump and probe pulses. From the definition of the Verdet constant it follows that the frequency is proportional to the net magnetization of the medium. This is exactly what is observed in our experiment (Fig. 3 (a-d)). Moreover, using Equation (3), we calculated the Verdet constant $V_{+}$corresponding to the frequencies of the Faraday modulation at $B_{0}=10 \mathrm{kG}$ (Methods). The results are plotted in the inset of Fig. 3 (f). The calculated values are in a good agreement with $1 / \lambda^{2}$ dependence of the Verdet constant reported earlier ${ }^{20}$ (see line in inset of Fig. 3 (f)).

The phase of the oscillations strongly depends on both the pump polarization and the external magnetic field polarity (Supplementary materials E,F), which agrees with the proposed model (Methods). The measured amplitude of the ultrafast modulation depends linearly on the pump fluence (Supplementary materials C). We also checked that the delay time at which the signal is quenched and the frequency of the modulation are independent on the fluence.

The modulation of the Faraday effect can be achieved for any counter propagating pulses. The pump can modulate the Faraday rotation of the probe which arrives to the sample before the pump and reflected from the rear face of the crystal. In this case, measuring the Faraday rotation of the reflected probe at the negative time delay, one should observe the modulation signal. In fact, we observed this strong signal at the negative time delays that underpins our interpretation (see details in Supplementary material G).

Finally, we note that pump-probe magneto-optical experiments is one of the main tools in the rapidly developing but also often controversial research area of ultrafast magnetism. ${ }^{3,11}$ The effects of propagation reported here manifests itself as oscillations of the Faraday rotation, the frequency of which is a linear function of the net magnetization. As a result, these oscillations can be mistakenly assigned to a collective mode of a spin excitation. Therefore our work has also important implications for interpretations of pump-probe magneto-optical experiments in transparent media.

${ }^{1}$ Schubert, O. et al. Sub-cycle control of terahertz high-harmonic generation by dynamical bloch oscillations. Nat Photon $\mathbf{8}, 119$ 123 (2014).

${ }^{2}$ Bigot, J.-Y. Femtosecond magneto-optical processes in metals. Comptes Rendus de l'Académie des Sciences - Series $\{I V\}$ Physics 2, 1483 - 1504 (2001).

${ }^{3}$ Bigot, J.-Y., Vomir, M. \& Beaurepaire, E. Coherent ultrafast magnetism induced by femtosecond laser pulses. Nat Phys $\mathbf{5}$, 515-520 (2009).

${ }^{4}$ Kirilyuk, A., Kimel, A. V. \& Rasing, T. Ultrafast optical manipulation of magnetic order. Rev. Mod. Phys. 82, 2731-2784 (2010).

${ }^{5}$ Kruglyak, V. V., Demokritov, S. O. \& Grundler, D. Magnonics. J. Phys. D: Appl. Phys. 43, 264001 (2010).

${ }^{6}$ Vogel, M. et al. Optically reconfigurable magnetic materials. Nat Phys 11, 487-491 (2015).

${ }^{7}$ Kumar, N. et al. Thz near-field faraday imaging in hybrid metamaterials. Opt. Express 20, 11277-11287 (2012).

${ }^{8}$ Riordan, J. A., Sun, F. G., Lu, Z. G. \& Zhang, X.-C. Freespace transient magneto-optic sampling. Appl. Phys. Lett. 71, 1452-1454 (1997).

${ }^{9}$ Kampfrath, T. et al. Coherent terahertz control of antiferromagnetic spin waves. Nat Photon 5, 31-34 (2011).

${ }^{10} \mathrm{Kimel}, \mathrm{A}$. V. et al. Ultrafast non-thermal control of magnetization by instantaneous photomagnetic pulses. Nature 435, 655-657 (2005).

${ }^{11}$ Satoh, T. et al. Writing and reading of an arbitrary optical polarization state in an antiferromagnet. Nat Photon 9, 25-29 (2015).

${ }^{12}$ Goto, T. et al. Optical tamm states in one-dimensional magnetophotonic structures. Phys. Rev. Lett. 101, 113902 (2008).

${ }^{13}$ Inoue, M., Levy, M. \& Baryshev, A. V. (eds.) Magnetophotonics (From Theory to Applications), vol. 1782013 of Springer Series in Materials Science (Springer, 2013).

${ }^{14}$ Thomsen, C., Grahn, H., Maris, H. \& Tauc, J. Surface generation and detection of phonons by picosecond light pulses. Phys. Rev. B 34, 4129-4138 (1986). 
${ }^{15}$ Scherbakov, A. V. et al. Coherent Magnetization Precession in Ferromagnetic $(\mathrm{Ga}, \mathrm{Mn})$ As Induced by Picosecond Acoustic Pulses. Phys. Rev. Lett. 105, 117204 (2010).

${ }^{16}$ Kim, J.-W., Vomir, M. \& Bigot, J.-Y. Ultrafast Magnetoacoustics in Nickel Films. Phys. Rev. Lett. 109, 166601 (2012).

${ }^{17}$ Kim, J.-W., Vomir, M. \& Bigot, J.-Y. Controlling the Spins Angular Momentum in Ferromagnets with Sequences of Picosecond Acoustic Pulses. Sci. Rep. 5, 8511- (2015).

${ }^{18}$ Popov, S. V., Svirko, Y. P. \& Zheludev, N. I. Pump-probe reflective polarization-sensitive nonlinear optics. J. Opt. Soc. Am. B 13, 2729-2738 (1996).

${ }^{19}$ Yasuhara, R. et al. Cryogenic temperature characteristics of verdet constant on terbium gallium garnet ceramics. Opt. Express 15, 11255-11261 (2007).

${ }^{20}$ Villora, E. G. et al. Faraday rotator properties of $\mathrm{Tb}_{3}\left(\mathrm{Sc}_{1.95} \mathrm{Lu}_{0.05}\right) \mathrm{Al}_{3} \mathrm{O}_{12}$, a highly transparent terbium-garnet for visible-infrared optical isolators. Appl. Phys. Lett. 99, 011111 (2011).

${ }^{21}$ Gruber, J. B. et al. Analyses of the optical and magneto-optical spectra of $\mathrm{Tb}_{3} \mathrm{Ga}_{5} \mathrm{O}_{12}$. J. Appl. Phys. 101, 023108 (2007).

${ }^{22}$ Mikhaylovskiy, R. V., Hendry, E., Ogrin, F. Y. \& Kruglyak, V. V. Low-temperature time-domain terahertz spectroscopy of terbium gallium garnet crystals. Phys. Rev. B 87, 094414 (2013).

${ }^{23}$ Löw, U. et al. Magnetization, magnetic susceptibility and ESR in $\mathrm{Tb}_{3} \mathrm{Ga}_{5} \mathrm{O}_{12}$. Eur. Phys. J. B 86, 87 (2013).

${ }^{24}$ Hurrell, J. P. et al. Optical phonons of yttrium aluminum garnet. Phys. Rev. 173, 851-856 (1968).

${ }^{25}$ Koningstein, J. A. \& Kane-Maguire, C. J. Electronic raman spectroscopy and magnetic susceptibilities. Can. J. Chem. 52, 3445-3450 (1974).

${ }^{26}$ Gavignet-Tillard, A., Hammann, J. \& De Seze, L. Crystal-field splitting of the fundamental multiplet of $\mathrm{Tb}^{3+}$ in terbium aluminum garnet. J. Phys. Chem. Solids 34, 241-248 (1973).

${ }^{27}$ Kamazawa, K. et al. Field-induced antiferromagnetism and competition in the metamagnetic state of terbium gallium garnet. Phys. Rev. B 78, 064412 (2008).

${ }^{28}$ Guillot, M. \& Marchand, A. Step-like magnetisation curves in $\mathrm{Tb}_{3} \mathrm{Ga}_{5} \mathrm{O}_{12}$. J. Phys. C 18, 3547 (1985).

${ }^{29}$ Kaganov, M., Pustyl'nik, N. \& Shalaeva, T. Magnons, magnetic polaritons, magnetostatic waves. Sov. Phys. Usp. 40 (1997).

${ }^{30}$ Stevens, T. E., Wahlstrand, J. K., Kuhl, J. \& Merlin, R. Cherenkov radiation at speeds below the light threshold: phononassisted phase matching. Science 291, 627-30 (2001).

\section{ACKNOWLEDGMENTS}

The authors acknowledge T. Satoh, D. Afanasev, A. Kirilyuk for valuable comments, A. Toonen for indispensable technical support. This work was supported by the European Community Seventh Framework Programme FP7-NMP-2011-SMALL-281043 (FEMTOSPIN), the European Research Council ERC Grant agreement No.257280 (Femtomagnetism), the
Foundation for Fundamental Research on Matter (FOM) as well as the Netherlands Organization for Scientific Research(NWO), program "Leading Scientist" of the Russian Ministry of Education and Science (14.z50.31.0034).

\section{AUTHOR CONTRIBUTION}

R.R.S., R.V.M., A.V.K. conceived the project. R.R.S. designed and built the experimental set up. R.R.S. and R.V.M. performed all measurements, analyzed the data. R.R.S., R.V.M., A.K.Z. developed the theoretical model with important suggestions from A.V.K.. R.R.S., R.V.M. and A.V.K co-wrote the paper with contributions from A.K.Z, Th. R. and V.V.K. The project was coordinated by A.V.K.

\section{COMPETING FINANCIAL INTERESTS}

The author(s) declare no competing financial interests.

\section{FIGURE LEGENDS}

1.

Geometry of the experiment and electronic structure of the $\mathbf{T b}^{3+}$ (a). (a) Experimental geometry used to measure the pump induced Faraday rotation of the transmitted probe. External magnetic field $\mathrm{B}_{0}$ is applied along [111] crystal axis, which coincides with the normal to the sample. Probe incidence is along the normal, while the pump incidence angle is $5^{\circ}$ from the normal. $\lambda / 2$, WP, BD are half wave plate, Wollaston prism and balanced photodiods, respectively. (b) Electronic structure of the $\mathrm{Tb}^{3+}$ ion in $\mathrm{Tb}_{3} \mathrm{Ga}_{5} \mathrm{O}_{12}{ }^{21}$ along with photon energies of the pump and probe pulses used in experiment. The photon energies of the probe pulse were $1.24,2.48,3.1 \mathrm{eV}$. The lowest states of the ground multiplet ${ }^{7} \mathrm{~F}_{6}$ of $\mathrm{Tb}^{3+}$ ion causing the main magnetic excitations are shown separately. $\Gamma_{i}$ represents states ${ }^{21}$ of the local group symmetry $\mathrm{D}_{2} . \Omega_{C F}$ is frequency of the electronic transition in the ground multiplet. 
2.

\section{Experimental observation of the ultrafast Fara-} day modulation. (a) Typical temporal response of the pump induced polarization rotation of the probe pulse at $B_{0}=5 \mathrm{kG}$ and $T=1.7 \mathrm{~K}$. The double arrow shows the limited time window of the observed oscillations. Inset shows temporal response of the pump induced transmissivity changes of the probe. Thick line shows calculated form of the temporal overlap in the vicinity of the $0 \mathrm{ps}$ (Methods). (b) FFT spectrum of the signal shown in panel (a) without the overlap region.

3.

The frequency of the magneto-optical modulation. (a) Transient Faraday rotation at different magnetic fields for $T=1.7 \mathrm{~K}$ (b) Magnetic field dependence of the frequencies of oscillations $\Omega / 2 \pi$ (Blue spheres). Black circles are for $\mathrm{Tb}^{3+}$ excitation. Lines are normalized sample magnetization vs magnetic field from Ref. 23 and 28 (Ref. A and B, respectively). The dashed line represents the slope of the curve corresponding to $g_{\text {eff }}=55$. (c) Transient Faraday rotation at different temperatures for $B_{0}=30 \mathrm{kG}$. (d) Temperature dependence of the modulation frequency at $\mathrm{B}_{0}=30 \mathrm{kG}$. Line is a guide to the eye. Inset shows the temperature dependence of the inverse frequency of the modulation on temperature (circles) and linear fit with the Curie-Weiss dependence (line). (e) Transient Faraday rotation for several probe wavelengths $\lambda$ at $B_{0}=20 \mathrm{kG}$ and $T=1.7 \mathrm{~K}$. (f) Magnetic field dependence of the modulation frequency for $\lambda$ equal to $0.4,0.5$ and $1 \mu \mathrm{m}$. Inset shows the wavelength dependence of the calculated Verdet constant at $B_{0}=10 \mathrm{kG}$ (balls) and that retrieved from Ref. 20 (line). The calculated values are in a good agreement with $1 / \lambda^{2}$ dependence (see Methods). The pump pulses were linearly polarized and had a fluence of $\mathrm{F}=10 \mathrm{~mJ} / \mathrm{cm}^{2}$.

4.

Scheme of the ultrafast magneto-optical modulation via counter-propagating laser pulses. The probe pulse initially linearly polarized along the $x$ axis enters the medium after the linearly polarized pump pulse and propagates along the $z$ axis. An external magnetic field is applied along the $z$ axis. At certain point the probe pulse encounters the back reflected pump pulse. The optical Kerr effect, resulting in acquiring additional polarization rotation by the probe pulse, depends on mutual orientation of polarization of the two counter propagating pulses at the moment of encounter defined by the time delay between them.

\section{METHODS}

\section{Experimental technique}

For the time-resolved magneto-optical studies we employed a Ti:sapphire regenerative amplifier laser system with a pulse width of $70 \mathrm{fs}$ and a repetition rate of $1 \mathrm{kHz}$. The incident pump and probe pulses were linearly polarized by Glan-Thompson polarizers. The relative orientation of the pump and probe polarization planes was controlled by achromatic half-wave plates. The magnetooptical measurements were performed on a $1 \mathrm{~mm}$ thick single crystal plate of undoped $\mathrm{Tb}_{3} \mathrm{Ga}_{5} \mathrm{O}_{12}$ cut perpendicularly to the (111)-plane. The sample was mounted in a superconducting split coil magneto-optical cryostat providing magnetic fields up to $70 \mathrm{kG}$ and temperatures down to $1.7 \mathrm{~K}$. The experiment was carried out in the transmission geometry with the magnetic field applied parallel to the [111] direction of the crystal. By changing the time delay between the pump and probe pulses, the pump induced polarization rotation and the transmissivity changes of the probe beam were measured. The probe beam incidence was along the sample normal. The incidence angle of the pump pulse was about 5 degrees away from the normal (Fig. 1 (a)). The wavelength of the pump pulse was set to $0.8 \mu \mathrm{m}$ (photon energy $1.55 \mathrm{eV})$, whereas the probe pulse wavelength was at $0.4 \mu \mathrm{m}, 0.5 \mu \mathrm{m}, 1 \mu \mathrm{m}$ (photon energy $3.1 \mathrm{eV}, 2.47 \mathrm{eV}$, $1.24 \mathrm{eV}$, respectively). Spot diameters of both beams were kept approximately at a size of $200 \mu \mathrm{m}$. In one set of the measurements rotation of the polarization plane and transmissivity changes of the probe beam were measured by means of a pair of balanced photo diodes. Signals were detected using a lock-in technique with the laser repetition rate as the reference frequency. 


\section{Temporal overlap width calculation in the thick medium}

To calculate the temporal overlap of the two laser pulses in the thick medium one has to make convolution not only in time but also in space:

$$
\begin{aligned}
& f(\tau)= \\
& =\int_{0}^{L} \int_{-\infty}^{+\infty} I_{p r}\left(t-\tau-\frac{z n_{p r}}{c_{0}}\right) I_{p u}\left(t-\frac{z n_{p u}}{c_{0}}\right) \mathrm{d} t \mathrm{~d} z,
\end{aligned}
$$

where $I_{p r}$ and $I_{p u}$ are envelopes of the optical intensities of probe and pump with a temporal width of $100 \mathrm{fs}, n_{p r}$ and $n_{p u}$ are the group refractive indices of the probe and pump pulses, accordingly. The result of the calculation for the Gaussian pulses by means of equation (4) is shown in the inset of Fig. 2 (a) by the orange line.

\section{Theoretical formalism for the magneto-optical modulation}

To derive the ultrafast Faraday modulation signal introduced in the manuscript we made use of a simple model accounting for the probe beam propagating through a counter propagating Kerr nonlinearity induced by the pump. The Kerr nonlinearity is linear dichroic region with respect to the probe. ${ }^{18}$ This results in the pump induced polarization rotation $\theta$ of the probe by the optical Kerr effect proportional to:

$$
\Theta(\tau) \sim I_{p} \sin (2 \Delta \phi)
$$

where $\Delta \phi$ is angle between linear polarization azimuths of the pump and probe pulses, $\tau$ is the time delay between the pump and probe pulses, $I_{p}$ is intensity of the pump pulse. The coordinate where the two pulses encounter inside the crystal ( $x=0$ is taken at the rear face of the crystal) is written:

$$
x=-\frac{L\left(n_{p r}-n_{p u}\right)}{\left(n_{p r}+n_{p u}\right)}+\frac{c_{0} \tau}{\left(n_{p r}+n_{p u}\right)} .
$$

Here $n_{p r}$ and $n_{p u}$ are the group refractive indices of the probe and pump pulses, respectively. Equation (6) allows us to estimate the time of the quench in experiment (equation (2)). The optical paths of the pump and probe pulses before encounter occurring in the crystal depend on the time delay between them. One can calculate that the these paths for the pump and probe pulses, respectively, are equal to:

$$
\begin{aligned}
\Delta x_{p u} & =\frac{2 L n_{p r}}{\left(n_{p r}+n_{p u}\right)}+\frac{c_{0} \tau}{\left(n_{p r}+n_{p u}\right)} \\
\Delta x_{p r} & =\frac{2 L n_{p u}}{\left(n_{p r}+n_{p u}\right)}-\frac{c_{0} \tau}{\left(n_{p r}+n_{p u}\right)} .
\end{aligned}
$$

Note that $\Delta x_{p u}+\Delta x_{p r}=2 L$. In magnetic field both pulses acquire a polarization rotation. The polarization rotation of the probe and pump pulses at the moment of the encounter are written as follows:

$$
\begin{aligned}
& \phi_{p r}=\Phi_{p r}-\frac{2 V_{p r} B_{0} L n_{p u}}{\left(n_{p r}+n_{p u}\right)}-V_{p r} B_{0} \frac{c_{0} \tau}{2 \bar{n}_{g}} \\
& \phi_{p u}=\Phi_{p u}+\frac{2 V_{p u} B_{0} L n_{p u}}{\left(n_{p r}+n_{p u}\right)}+V_{p u} B_{0} \frac{c_{0} \tau}{2 \bar{n}_{g}},
\end{aligned}
$$

where $\bar{n}_{g}=\left(n_{p u}+n_{p r}\right) / 2, L$ is the thickness of the sample, $\Phi_{p r}$ and $\Phi_{p u}$ are the initial polarization angles of the probe and pump, respectively, $V_{p r}$ and $V_{p u}$ are the Verdet constants at the wavelength of the probe and pump, respectively. Substituting $\Delta \phi=\phi_{p u}-\phi_{p r}$ from equation (8) to equation (5) one obtains probe polarization rotation for the fixed initial polarization of the probe $\Phi_{p r}=0$ :

$$
\begin{aligned}
& \Theta(\tau) \sim I_{p u} \sin 2 \Delta \phi= \\
& =I_{p u} \sin 2\left(\Phi_{p u}+L B_{0} V^{*}\right) \cos \left(\frac{B_{0} c_{0} V_{+} \tau}{\bar{n}_{g}}\right)- \\
& -I_{p u} \cos 2\left(\Phi_{p u}+L B_{0} V^{*}\right) \sin \left(\frac{B_{0} c_{0} V_{+} \tau}{\bar{n}_{g}}\right) .
\end{aligned}
$$

Here substitutions are made for $V_{+}=V_{p u}+V_{p r}$ and $V^{*}=4\left[n_{p u} V_{p u}+n_{p r} V_{p r}\right] /\left[n_{p r}+n_{p u}\right]$. From equation (9) one can find the frequency of the ultrafast modulation:

$$
\Omega=\frac{B_{0} c_{0} V_{+}}{\bar{n}_{g}}
$$

\section{The pump polarization dependence of the modulation}

It is seen from equation (9) that the signal contains two components $\cos (\Omega \tau)$ and $\sin (\Omega \tau)$ which depend differently on the pump polarization angle $\Phi_{p u}$. Supplementary materials $\mathrm{E}$ shows the probe modulation for different $\Phi_{p u}$ at $B_{0}=35 \mathrm{kG}$ and $T=1.7 \mathrm{~K}$. One sees that the amplitude of the modulation is unchanged while the phase changes with changing the pump polarization. To check whether the observed behavior is consistent with our model, we performed a fit of the time traces in Supplementary materials E as suggested by equation (9). One can see that this form describes perfectly 
the experimental data shown in Supplementary materials E. Indeed, the experimental signal is proportional to $\sin \left(\Phi_{p u}\right) \cos (\Omega \tau)+\cos \left(\Phi_{p u}\right) \sin (\Omega \tau)$.

\section{The Verdet constant calculation}

Equation (10) permits one to estimate the Verdet constant from our measurements:

$$
V_{+}=\frac{\Omega\left(n_{p r}+n_{p u}\right)}{2 B_{0} \cdot c_{0}} .
$$

Using the corresponding frequencies of the modulations at a magnetic field of $10 \mathrm{kG}$ for wavelengths of the probe of $0.4 \mu \mathrm{m}, 0.5 \mu \mathrm{m}$, and $1 \mu \mathrm{m}$, we calculated the Verdet constant $V_{+}$(see inset of Fig. $3(\mathrm{c})$ ). To compare the calculated values of the Verdet constant $V_{+}$we used the formula for the wavelength dependence of the Verdet constant from Ref. 20:

$$
V=\frac{E}{\lambda^{2}-\lambda_{0}^{2}}+V_{0}
$$

$E$ is a proportionality factor, $\lambda_{0}$ is the band-gap wavelength, $V_{0}$ is an offset value. We used the Curie-Weiss law to recalculate the parameter $E$ from the temperature of $300 \mathrm{~K}$ to $2 \mathrm{~K}$ in our experiment. The parameter was taken from Ref.20. Fixing the value $E$ at the temperature of $1.7 \mathrm{~K}$ and assuming $\lambda_{0}$ to be independent on temperature we fitted the experimentally calculated Verdet constants from equation (11) by equation 12 . The result of the fit in the inset of Fig. 3 (f) demonstrates a very good quantitative agreement. 
(a)

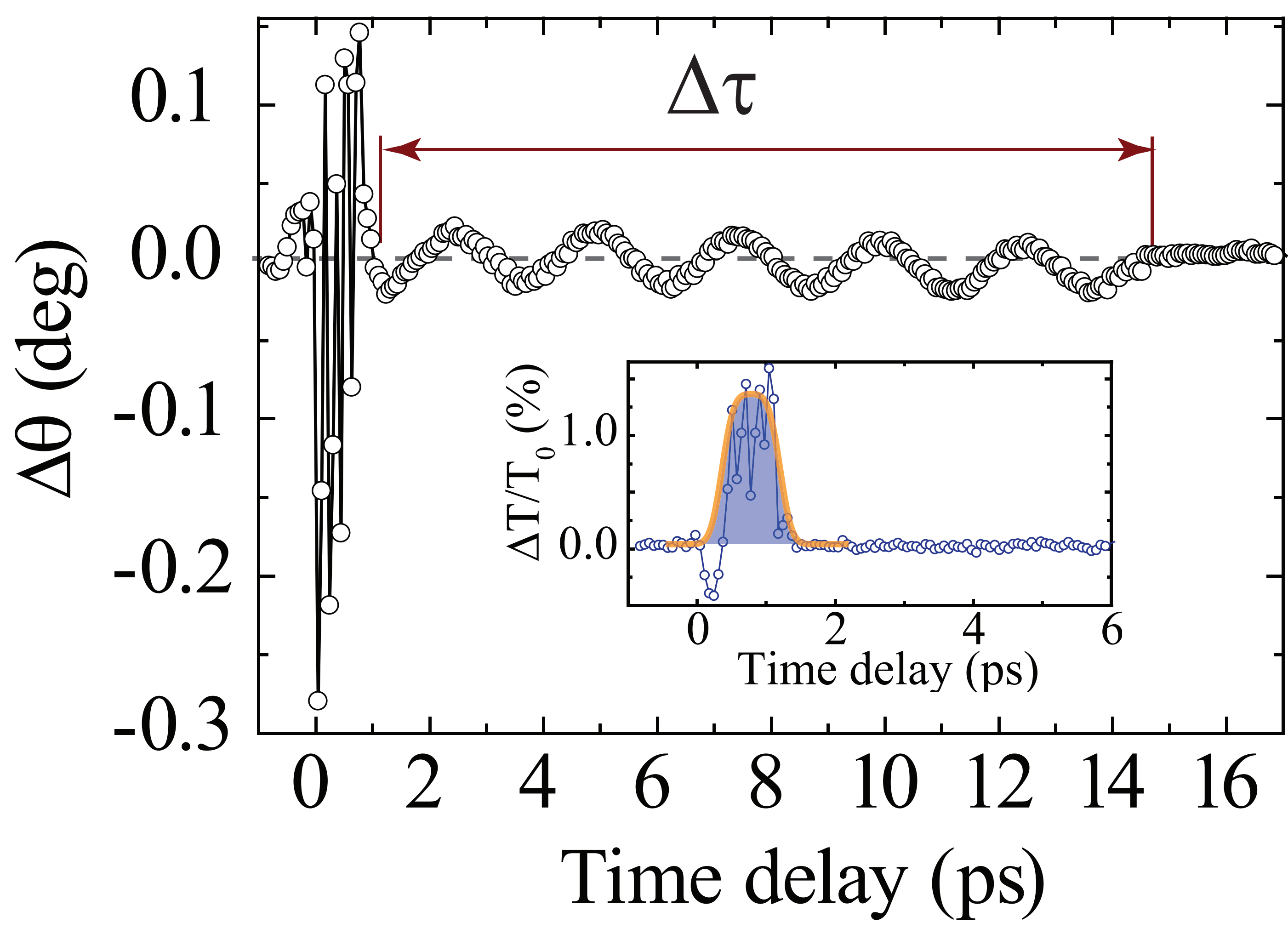

(b)

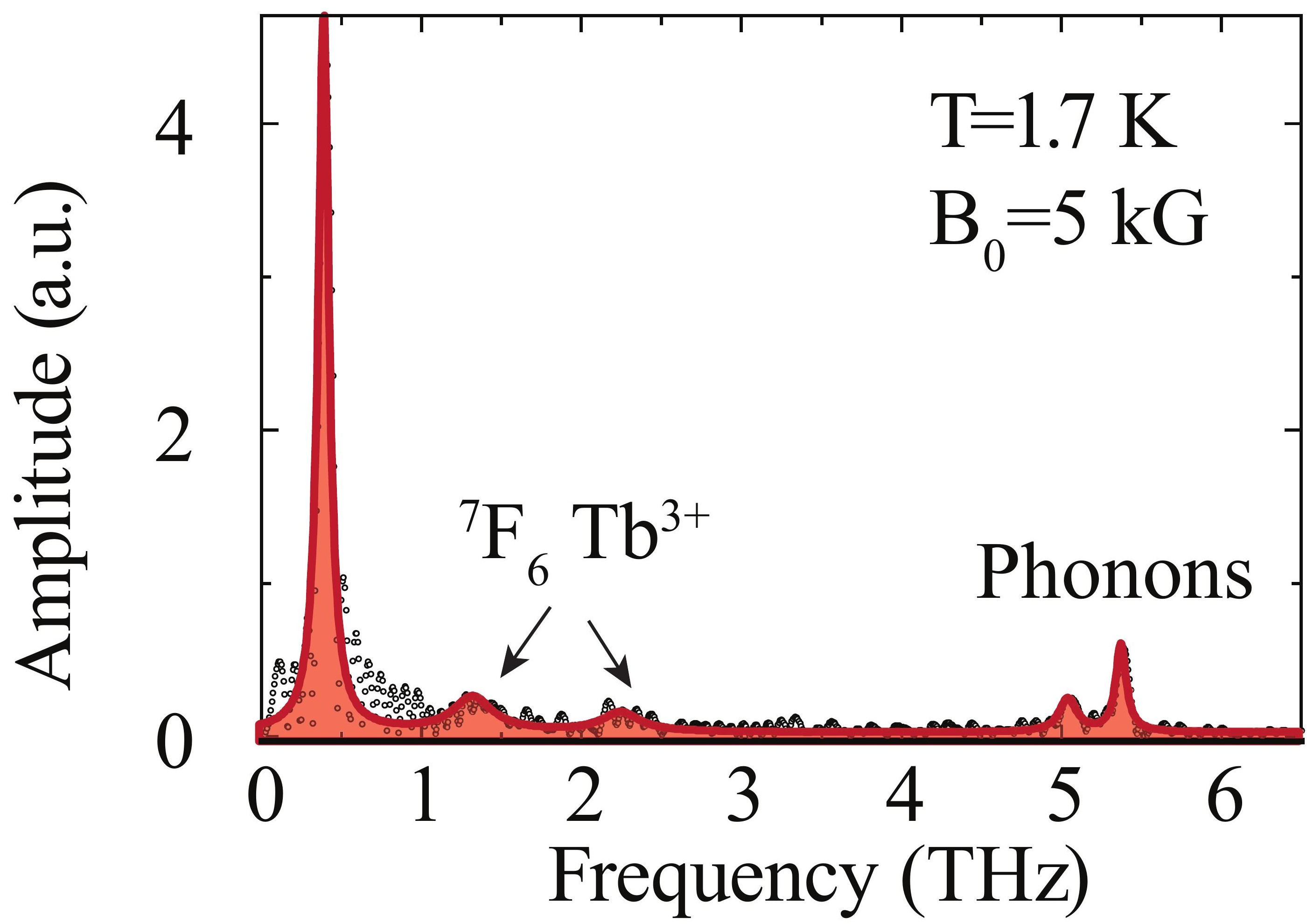




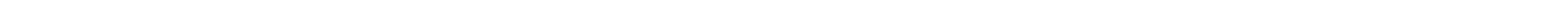

